\title{
The interaction between artemether- lumefantrine and lopinavir/ritonavir-based antiretroviral therapy in HIV-1 infected patients
}

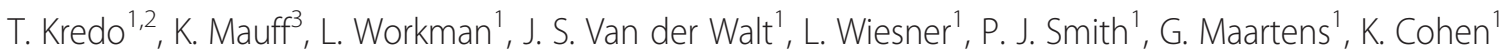
and K. I. Barnes ${ }^{1,4^{*}}$

\begin{abstract}
Background: Artemether-lumefantrine is currently the most widely recommended treatment of uncomplicated malaria. Lopinavir-based antiretroviral therapy is the commonly recommended second-line HIV treatment. Artemether and lumefantrine are metabolised by cytochrome P450 isoenzyme CYP3A4, which lopinavir/ ritonavir inhibits, potentially causing clinically important drug-drug interactions.

Methods: An adaptive, parallel-design safety and pharmacokinetic study was conducted in HIV-infected (malaria-negative) patients: antiretroviral-naïve and those stable on lopinavir/ritonavir-based antiretrovirals. Both groups received the recommended six-dose artemether-lumefantrine treatment. The primary outcome was day-7 lumefantrine concentrations, as these correlate with antimalarial efficacy. Adverse events were solicited throughout the study, recording the onset, duration, severity, and relationship to artemether-lumefantrine. Results: We enrolled 34 patients. Median day-7 lumefantrine concentrations were almost 10-fold higher in the lopinavir than the antiretroviral-naïve group [3170 versus $336 \mathrm{ng} / \mathrm{mL} ; p=0.0001$ ], with $A \cup C_{(0-i n f)}$ and $C_{\max }$ increased five-fold [2478 versus $445 \mu \mathrm{g} . \mathrm{h} / \mathrm{mL} ; p=0.0001$ ], and three-fold [28.2 versus $8.8 \mu \mathrm{g} / \mathrm{mL} ; p<0.0001$ ], respectively. Lumefantrine $C_{\max }$, and $A \cup C_{(0-i n f)}$ increased significantly with $\mathrm{mg} / \mathrm{kg}$ dose in the lopinavir, but not the antiretroviral-naïve group. While artemether exposure was similar between groups, $C_{\max }$ and $A \cup C_{(0-8 h)}$ of its active metabolite dihydroartemisinin were initially two-fold higher in the lopinavir group $[p=0.004$ and $p=0.0013$, respectively]. However, this difference was no longer apparent after the last artemether-lumefantrine dose. Within 21 days of starting artemether-lumefantrine there were similar numbers of treatment emergent adverse events (42 vs. 35 ) and adverse reactions (12 vs. 15, $p=0.21$ ) in the lopinavir and antiretroviral-naïve groups, respectively. There were no serious adverse events and no difference in electrocardiographic QTcF-and PR-intervals, at the predicted lumefantrine $T_{\max }$.

(Continued on next page)
\end{abstract}

\footnotetext{
* Correspondence: karen.barnes@uct.ac.za

'Division of Clinical Pharmacology, Department of Medicine, University of Cape Town, Cape Town, South Africa

${ }^{4}$ WorlldWide Antimalarial Resistance Network (WWARN), Oxford, UK

Full list of author information is available at the end of the article
} 
(Continued from previous page)

Conclusion: Despite substantially higher lumefantrine exposure, intensive monitoring in our relatively small study raised no safety concerns in HIV-infected patients stable on lopinavir-based antiretroviral therapy given the recommended artemether-lumefantrine dosage. Increased day-7 lumefantrine concentrations have been shown previously to reduce the risk of malaria treatment failure, but further evidence in adult patients co-infected with malaria and HIV is needed to assess the artemether-lumefantrine risk : benefit profile in this vulnerable population fully. Our antiretroviral-naïve patients confirmed previous findings that lumefantrine absorption is almost saturated at currently recommended doses, but this dose-limited absorption was overcome in the lopinavir group.

Trial registration: Clinical Trial Registration number NCT00869700. Registered on clinicaltrials.gov 25 March 2009

Keywords: HIV, Malaria, Artemether, Lumefantrine, Lopinavir, Ritonavir, Drug interaction, Safety, Pharmacokinetic, Dose-related exposure

\section{Background}

With the overlapping geographic distribution of HIV and $P$. falciparum malaria, many patients may require co-treatment with antiretrovirals and antimalarials. For uncomplicated malaria, the World Health Organization (WHO) recommends artemisinin-based combination therapies (ACTs), of which the fixed-dose combination artemether-lumefantrine is currently most widely used, accounting for $73 \%$ of ACTs procured in 2013 [1]. The precise pharmacokinetic determinants of treatment outcome in uncomplicated malaria remain uncertain, but the area under the concentration-time curve (AUC) and the concentration on day- 7 of slowly eliminated antimalarials are considered important predictors [2, 3]. The 'therapeutic' day-7 lumefantrine concentrations published to date range between $170 \mathrm{ng} / \mathrm{mL}$ to $500 \mathrm{ng} / \mathrm{mL}$, with a concentration of $280 \mathrm{ng} / \mathrm{mL}$ most often cited [4-12].

As the HIV pandemic matures, increasing numbers of patients develop resistance to first-line antiretroviral therapy (ART) and are placed on second-line ART. Ritonavir-boosted lopinavir-based ART is the most widely used second-line ART in Africa and South East Asia. Artemether, lumefantrine and lopinavir are all primarily metabolised by the same cytochrome $\mathrm{P} 450$ (CYP) iso-enzyme, CYP3A4. Ritonavir is a potent inhibitor of CYP3A4 creating the potential for clinically significant drug-drug interactions [13]. Although the interaction between lopinavir and ritonavir is used for therapeutic advantage, known as 'boosting, there is limited evidence to inform clinicians and policy makers about the interaction between artemether-lumefantrine and lopinavirbased ART, leading to inconsistent recommendations on the use of artemether-lumefantrine in patients co-infected with HIV/AIDS [12, 14, 15]. As access to antiretrovirals and ACTs increase, the importance of defining the interaction between antimalarials and ART becomes more urgent. Our study investigated the pharmacokinetics and safety of the recommended adult dose of artemether- lumefantrine when given to HIV-infected patients stable on lopinavir-based ART.

\section{Methods \\ Subjects and study design}

We conducted a sequential, two-period, adaptive design, open-label, pharmacokinetic and safety drug-drug interaction study at the Groote Schuur Hospital Clinical Pharmacology Research Ward in Cape Town, South Africa.

HIV-infected adults (18 years of age or older) with CD4+ lymphocyte counts greater than 200 cells $/ \mu \mathrm{L}$ were enrolled. Participants enrolled were stable on treatment with lopinavir-based ART for a minimum of six weeks. They were compared with a group of patients who were antiretroviral (ARV)-naïve and not yet eligible for ART, according to the South African National HIV Treatment Guidelines at the time [16, 17]. The participants were otherwise well adults without renal disease and were not geriatric, underweight, overweight or obese [18].

Exclusion criteria for safety reasons were a current diagnosis of malaria, known hypersensitivity to artemether or lumefantrine, pregnancy (as confirmed by a serum Beta-HCG test), breast-feeding, or clinically relevant hepatic or renal dysfunction. In addition, those with a pre-existing (or family history of) prolonged QT interval, cardiac dysrhythmia, electrolyte disturbances or taking any drugs known to prolong the QT interval, were excluded. Exclusion criteria for potential confounding of the pharmacokinetic parameters included participants using other drugs known to interact via the CYP450 enzyme system, current smokers, or alcohol users who would not abstain from alcohol intake for the trial duration. Caffeine, grapefruit juice or strenuous exercises were not permitted from $24 \mathrm{~h}$ before and during study admission.

\section{Ethics, consent and permissions}

Patients provided written informed consent prior to enrollment. Regulatory approval was received from 
the University of Cape Town Research Ethics Committee and the South African Medicines Control Council (Clinical Trial Registration number NCT00869700). The procedures followed were in accordance with the Good Clinical Practice Guidelines, including the Helsinki Declaration of 1975, as revised in 2008.

\section{Dosing and pharmacokinetic sample collection}

As there was a safety concern about increases in lumefantrine concentrations secondary to inhibition by ritonavir and lopinavir, patients on lopinavir-based treatment were admitted for a single dose of artemether-lumefantrine $(80 \mathrm{mg} / 480 \mathrm{mg})$ in a dosefinding safety phase. Pharmacokinetic and safety results were analysed and reviewed by the Data Safety Monitoring Board prior to approval of the adapted dose used in the multiple-dosing phase. The ARV-naïve participants took part in the multiple-dosing phase only, when the recommended adult $80 \mathrm{mg} / 480 \mathrm{mg}$ artemether-lumefantrine dose was given at $0,8,24$, 36, 48 and $60 \mathrm{~h} \mathrm{[12].}$

In both study groups, all doses were administered with $40 \mathrm{~mL}$ of soya milk ( $0.8 \mathrm{~g}$ fat) and a meal containing a minimum of $6 \mathrm{~g}$ of fat within one hour of each dose, with the exception of dose 2 (at $8 \mathrm{~h}$ ) when only soya milk accompanied the dose.

Participants were admitted for rich pharmacokinetic sampling (until $72 \mathrm{~h}$ after the first artemetherlumefantrine dose). Subsequent samples were collected on an outpatient basis until day 21 . Venous blood samples were collected into heparinised (LH PST II) BD Vacutainer $^{\ominus}$ tubes. The blood tubes were pre-chilled on ice for $10 \mathrm{~min}$; all samples were again chilled before being centrifuged at $4{ }^{\circ} \mathrm{C}$ for $10 \mathrm{~min}$ at $2000 \mathrm{~g}$. The resulting plasma was stored at $-80{ }^{\circ} \mathrm{C}$ within 30 min of the blood draw. Pharmacokinetic assays were done within four months of sample collection.

For the Phase 1 (single-dose) pharmacokinetic profile: Plasma concentrations of lumefantrine were assayed at pre-dose (0 h), $0.5,1,1.5,2,3,4,5,6,8,14,24,36,48$, $60,72,96,120,144,168,336$ and $504 \mathrm{~h}$ post and artemether/dihydroartemisinin concentrations were assayed at pre-dose $(0 \mathrm{~h}), 0.5,1,1.5,2,3,4,5,6,8$, and $24 \mathrm{~h}$ after the first artemether-lumefantrine dose.

For the Phase 2 (full-treatment dose) pharmacokinetic profile: Plasma concentrations of lumefantrine were assayed at pre-dose $(0 \mathrm{~h}), 0.5,1,1.5,2,3,4,5$, $6,8,14,24,30,36,42,48,54,60,61.5,62,63,64$, $65,66,68,70,72,96,120,144,168,336$ and $504 \mathrm{~h}$, and artemether/dihydroartemisinin concentrations were assayed at pre-dose $(0 \mathrm{~h}), 0.5,1,1.5,2,3,4,5,6,8$, $24,60,61.5,62,63,64,65,66,68,70$ and 72 h postdose.

\section{Pharmacokinetic assays}

Concentrations of lumefantrine, artemether, and dihydroartemisinin were determined by the Division of Clinical Pharmacology Laboratory, University of Cape Town using validated liquid chromatography tandem mass spectrometer (LC-MS/MS) assays as described previously [16].

\section{Safety data collection}

A clinical evaluation and full-blood count, renal function tests, liver enzymes, lactate and glucose blood tests were performed at screening and at the final safety visit 21 days after the first artemether-lumefantrine dose in both the single-dose and multiple-dose phases of the study. CD4+ lymphocyte counts and HIV-1 viral loads and serum pregnancy tests (in all women) as well as urine tests for drugs of abuse (amphetamines, benzodiazepines and opiates) were performed at screening. Twelve-lead single electrocardiograms (ECGs) were performed at screening, pre-dose and at the expected time of maximal lumefantrine plasma concentration $(68 \mathrm{~h}$ post-dose) [19]. An independent cardiologist assessed all ECGs and the QT interval was corrected using the Fridericia formula [20]. Adverse events were solicited throughout the study, starting on completion of screening and recording the onset, duration, severity, relationship to study drug and need for treatment $[21,22]$. These were classified using MedDRA preferred terms. Some participants in the lopinavir group were also included in a methods sub-study evaluating more intensive methods for eliciting adverse event data from participants including checklists, in-depth interviews and focus group discussions [22].

\section{Statistical methods}

The sample size was calculated to demonstrate a 2-fold change in lumefantrine exposure (day 7 concentration or AUC), i.e., such that the $90 \%$ confidence intervals (CIs) for geometric mean ratios lie outside the interval 0.5 to 2.0 with a power of $80 \%$ [19]. Thirteen participants were required in each group and a total of 18 participants were recruited for each arm to accommodate potential dropouts [16].

Data analysis and pharmacokinetic modelling (noncompartmental) were performed using Stata 13 (StataCorp, College Station, Texas). Concentrations below the limits of quantification were considered missing. Area under the concentration-time curve $\left(\mathrm{AUC}_{0-\infty}\right)$ was calculated using the trapezoidal rule. Elimination half-life was calculated as $\ln (2) / \lambda z$, where $\lambda z$ is the first order rate constant associated with the terminal (log-linear) portion of the curve, estimated by linear regression of time vs. log concentration, using the default of last three data points. 
In order to predict a safe dose for administration in Phase 2, the lumefantrine concentration-time data $(0-8 \mathrm{~h})$ from our single-dose safety phase (Phase 1 ) were compared with those in 18 ARV-naïve subjects included in our prior antimalarial-antiretroviral drug interaction study using geometric mean ratios [16]. In the latter study the subjects completed a full course of artemether-lumefantrine using the same schedule as in the multiple-dose phase.

Determinants of lumefantrine day-7 concentrations, $\mathrm{AUC}_{(0-\mathrm{inf})}$ and $\mathrm{C}_{\max }$ values were explored using linear regression of the log transformed values, with results reported as geometric mean ratios (GMR). The Spearman rank correlation test was used to test the correlation between lumefantrine day-7 concentrations and $\mathrm{AUC}_{(0-\text { inf })}$. Logistic regression was used to explore the determinants of day-7 lumefantrine concentrations below the reported therapeutic concentration $(280 \mathrm{ng} / \mathrm{mL})$. Continuous and categorical covariates were compared between groups at baseline using Kruskal-Wallis and Chi-squared tests, respectively. Kruskall Wallis tests were also used for simple comparisons of the day-7 lumefantrine concentrations, $\mathrm{AUC}$ and $\mathrm{C}_{\max }$ values between groups. In order to account for the repeated measures per subject, particularly given previously reported auto-induction effects with the artemisinins, mixed-effect regression models were used to assess the possible impact of dose-occasion on artemether and dihydroartemisinin exposure, where the responses were log-transformed $\mathrm{AUC}$ and $\mathrm{C}_{\max }$ values.

Secondary safety endpoints included frequency and severity of adverse events, changes in haematological, serum biochemical and urinalysis parameters, and vital signs between screening and follow-up. The risk of adverse drug reactions was compared between treatment groups using logistic regression. ECG parameters (PR-, QRS-, RR- and QT-intervals) were compared within groups between screening and the predicted lumefantrine $\mathrm{T}_{\max }$ using the Wilcoxon signed rank test, while the Wilcoxon rank sum test was used to compare these between groups and within period, and their correlation with lumefantrine concentrations was assessed using the Spearman Rank correlation test.

\section{Results}

\section{Study population}

Thirty-six adults (18 in the ARV-naïve group and 18 in the lopinavir group) were recruited. All 18 in the ARVnaïve group and 16/18 (89 \%) in the lopinavir group completed the study. One participant was replaced after being excluded prior to Phase 2 dosing due to starting a potentially interacting medication (amitriptyline); two participants withdrew consent during phase 2 (one to attend a funeral in another province, the other provided no explanation). At baseline, the groups were well matched for weight-adjusted $(\mathrm{mg} / \mathrm{kg})$ lumefantrine dose and CD4+ lymphocyte count. ARV-naïve patients were younger $(p=0.0001)$, and had a higher median viral load (3.76 $\log _{10}$ copies $\left./ \mathrm{mL} ; p=0.0005\right)$. The mean corpuscular volume was higher with ARV use, as is expected with AZT and d4T. Gamma glutamyl-transferase levels were higher in the lopinavir group than the ARV-naïve group (median 26 (IQR 18-44) U/L vs. $15(13-29) \mathrm{U} / \mathrm{L} ; p=0.03)$, which reflects the higher upper limit of the normal range in males than females (60 vs. $35 \mathrm{U} / \mathrm{L}$ ); males made up $6 \%$ of the ARV-naïve group and $31 \%$ of the lopinavir group. Six participants in the ARV-naïve group and five in the lopinavir group $(p=1.0)$ were receiving cotrimoxazole prophylaxis (Table 1 ).

\section{Pharmacokinetic results}

Single-dose safety phase (Phase 1)

The non-compartmental analysis of the single artemetherlumefantrine dose, safety phase (Phase 1) in the lopinavir group was compared with the ARV-naïve group. The GMR $(90 \% \mathrm{CI})$ for the lumefantrine $\mathrm{C}_{\max }$ was 1.86 (1.48-2.33), while that for the lumefantrine $\mathrm{AUC}_{(0-8 \mathrm{~h})}$ was 1.78 (1.43-2.33). The Data Safety Monitoring Board and investigators agreed based on the predefined criteria (i.e., GMR between 0.5 and 2) to continuing to Phase 2 using the full recommended adult six-dose artemether-lumefantrine regimen.

\section{Effect of lopinavir-based ART on lumefantrine plasma concentrations following six-dose artemether-lumefantrine regimen (Phase 2)}

The lumefantrine plasma concentration-time curves (0-504 hours) are depicted in Fig. 1 and summarised in Table 2. Median lumefantrine maximum $\left(C_{\max }\right)$ concentrations of 8.76 and $28.15 \mu \mathrm{g} / \mathrm{mL}$ were achieved at a median time $\left(\mathrm{T}_{\max }\right)$ of 42 and $67 \mathrm{~h}$ respectively in the ARV-naïve and lopinavir groups. The median area under the plasma lumefantrine concentration time curve $\left(\mathrm{AUC}_{(0 \text {-inf })}\right)$ was $2478 \mu \mathrm{g} . \mathrm{h} / \mathrm{mL}$ in the lopinavir group and $445 \mu \mathrm{g} . \mathrm{h} / \mathrm{mL}$ in the ARVnaïve group $(p=0.0001)$. Elimination half-life also appeared longer in the lopinavir group (4.6 vs $4.1 \mathrm{~h}$, $p=0.0027$ ), although this is more likely to reflect improved bioavailability with lopinavir co-administration.

Median (range) day-7 lumefantrine concentrations were $3170(772-18,100) \mathrm{ng} / \mathrm{mL}$ in the lopinavir group compared to $336(29-934) \mathrm{ng} / \mathrm{mL}$ in the ARV-naïve group $(p=0.0001)$. Across both groups, each $1 \mathrm{mg} / \mathrm{kg}$ increase in the lumefantrine dose increased day-7 lumefantrine concentrations by $5.9 \%$ (95\% CI 2.1-9.8\%; $p=$ 0.003). After adjusting for $\mathrm{mg} / \mathrm{kg}$ dose, the lumefantrine day-7 concentration for the lopinavir group was 10 -fold 
Table 1 Baseline characteristics in HIV-1 infected patients who are antiretroviral-naïve $(n=18)$ or on lopinavir-based antiretroviral therapy $(n=16)$

\begin{tabular}{|c|c|c|c|}
\hline Parameter & ARV-Naïve group & Lopinavir group & $p$ value \\
\hline Sex female n (\%) & $17(94 \%)$ & $11(69 \%)$ & 0.078 \\
\hline Age (years) & $27(25-32)$ & $37(33-41)$ & $<0.0001$ \\
\hline Total lumefantrine dose (mg/kg) & $49.7(43.0-52.4)$ & $46.5(41.4-51.9)$ & 0.72 \\
\hline Weight (kg) & $58(55-67)$ & $62(56-70)$ & 0.72 \\
\hline Albumin $(\mathrm{g} / \mathrm{L})$ & $40(39-44)$ & $43(41.5-44)$ & 0.14 \\
\hline Alkaline phosphatase $(\mathrm{U} / \mathrm{L})$ & $57.5(50-69)$ & $69.5(59-88)$ & 0.09 \\
\hline Gamma glutamyltransferase (U/L) & $40(39-44)$ & $43(42-44)$ & 0.03 \\
\hline Alanine transaminase $(\mathrm{U} / \mathrm{L})$ & $15(13-29)$ & $26(18-44)$ & 0.72 \\
\hline Aspartate transaminase (U/L) & $22(14-28)$ & $19(17-30)$ & 0.59 \\
\hline Mean corpuscular volume (fL) & $86.7(85-91)$ & $100.95(98-110)$ & 0.0001 \\
\hline Concomitant cotrimoxazole n (\%) & $6(33 \%)$ & $5(31 \%)$ & 1.0 \\
\hline CD4+ count $\left(\times 10^{6} / \mathrm{L}\right)$ & $356(260-507)$ & $375(296-590)$ & 0.7 \\
\hline HIV viral load (copies/mL) & $\log _{10} 3.76(3.0-4.1)$ & $<50$ copies/ $/ \mathrm{L}$ & 0.0005 \\
\hline
\end{tabular}

Values are shown as medians (interquartile ranges [IQRs]) or $\mathrm{n}(\%)$. Statistical significance ( $\mathrm{p}$ values) calculated using the Kruskal-Wallis test or chi-squared test, as appropriate

those in the ARV-naïve group (adjusted GMR 10.4 [95\% CI 6.4-16.9]; $p<0.0001$ ). None of the subjects in the lopinavir group had day-7 lumefantrine concentrations below a therapeutic threshold $(280 \mathrm{ng} / \mathrm{mL})$, compared to one-third (6/18) of those in the ARV-naïve group ( $p$ $=0.02)$. Lumefantrine day- 7 concentrations and $\mathrm{AUC}_{(0-}$ inf) were highly correlated (R-squared $=0.98)$.

Adjusting for $\mathrm{mg} / \mathrm{kg}$ dose (the only significant covariate), the median lumefantrine $\mathrm{AUC}_{(0 \text {-inf) }}$ was almost five-fold higher (GMR $4.8295 \%$ CI 3.41-6.79, $p<0.0001)$ and the median $C_{\max }$ values more than doubled (GMR $2.6795 \%$ CI 1.99-3.59; $p<0.0001$ ) in the lopinavir than the ARV-naïve group. However, the increase in exposure with $\mathrm{mg} / \mathrm{kg}$ dose was only seen

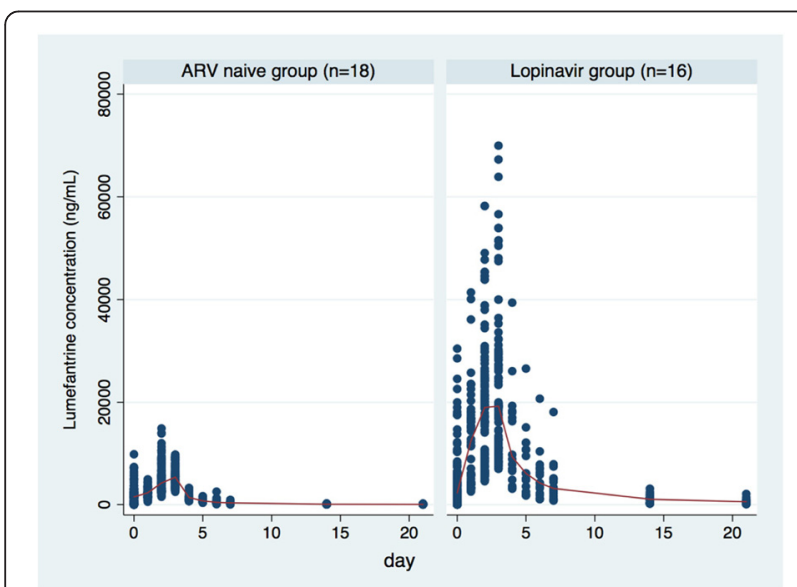

Fig. 1 Scatter plot of Plasma lumefantrine concentrations over time, by study group in the lopinavir group in whom the $\mathrm{C}_{\max }$ increased by $4.8 \%(95 \% \mathrm{CI} 0.9-8.6 \% ; p=0.019)$ and the $\mathrm{AUC}_{(0-\mathrm{inf})}$ increased by $5.4 \%(95 \% \mathrm{CI} 0.7-10.0 \%$; $p=0.026)$, respectively with each $1 \mathrm{mg} / \mathrm{kg}$ increase in lumefantrine dose (Fig. 2).

\section{Effect of lopinavir-based ART on artemether and dihydroartemisinin plasma concentrations following six-dose artemether-lumefantrine regimen (Phase 2)}

The artemether and dihydroartemisinin plasma concentration-time curves after artemether-lumefantrine dose one ( $0-8$ hours) and dose six (60-68 hours) are depicted in Fig. 3, with artemether and dihydroartemisinin pharmacokinetic parameters summarised by treatment group in Table 3.

For artemether, there were no significant differences between treatment groups for any of the pharmacokinetic parameters at either of the time periods studied, 0 to $8 \mathrm{~h}$ and 60 to $68 \mathrm{~h}$ (Table 3). However, the mixedeffect model showed a significant dose-occasion effect on the artemether $\mathrm{AUC}$ and $\mathrm{C}_{\max }$ in both treatment groups. After the last artemether-lumefantrine dose (60-68 hours), artemether $C_{\max }$ was $76 \%$ lower (GMR 0.24 [95 \% CI 0.17-0.34]) and AUC was $58 \%$ lower (GMR 0.42 [95 \% CI $0.30-0.59$ ]; $p<0.0001$ ) than after the first artemether-lumefantrine dose.

For dihydroartemisinin, after dose 1 ( 0 to $8 \mathrm{~h}$ ), the lopinavir group had almost double the exposure of the ARVnaïve group (median [range] $\mathrm{C}_{\max }$ of 77.5 [30.3-189.0] vs. $42.2[17.4-94.4] \mathrm{ng} / \mathrm{mL} ; p=0.004)$ and $\mathrm{AUC}_{(0-8 \mathrm{~h})}$ of 283.6 [110.1-495.6] vs. 123.8 [79.7-340.0] ng.h $/ \mathrm{mL} ; p=0.001$ ). By 60 to $68 \mathrm{~h}$ (after dose 6), exposure was similar between treatment groups other than slight differences in the 
Table 2 Lumefantrine pharmacokinetic parameters following six-dose artemether-lumefantrine treatment in HIV-1 infected patients who are antiretroviral-naïve or on lopinavir-based antiretroviral therapy

\begin{tabular}{llll}
\hline Parameter median (IQR) & ARV-naïve group $(n=18)$ & Lopinavir group $(n=16)$ & $p$ value \\
\hline$C_{\max }(\mu \mathrm{g} / \mathrm{mL})$ & $8.76(7.80-9.84)$ & $28.15(14.00-32.95)$ \\
$\mathrm{CV}$ & $26.3 \%$ & $59.6 \%$ & $67(51-70)$ \\
$\mathrm{T}_{\max }(\mathrm{h})$ & $42(42-66)$ & $27.1 \%$ \\
$\mathrm{CV}$ & $35.5 \%$ & $2478(1093-3596)$ \\
$\mathrm{AUC}_{(0-\mathrm{inf})}(\mu \mathrm{g} . \mathrm{h} / \mathrm{mL})$ & $445(357-553)$ & $71.0 \%$ \\
$\mathrm{CV}$ & $33.5 \%$ & $4.6(4.4-5.2)$ \\
$\mathrm{T}_{1 / 2}$ (days) & $4.1(2.7-4.4)$ & $15.4 \%$ \\
$\mathrm{CV}$ & $25.7 \%$ & $3170(1440-5085)$ \\
Day-7 conc $(\mathrm{ng} / \mathrm{mL})$ & $336(230-396)$ & $98.5 \%$ & $<0.0001$ \\
$\mathrm{CV}$ & $54.0 \%$ & $<0.0001$
\end{tabular}

Values are shown as medians (interquartile ranges [IQRs]) and Coefficient of variation (CV, \%). Cmax, maximal concentration; Tmax, time at the maximal concentration; $A \cup C(0-i n f)$, area under the plasma concentration-time curve, from $0 \mathrm{~h}$ to infinity; $t 1 / 2$, elimination half-life; Day- 7 concentration, lumefantrine concentration on day-7;. Statistical significance ( $p$ values) calculated using the Kruskal-Wallis test

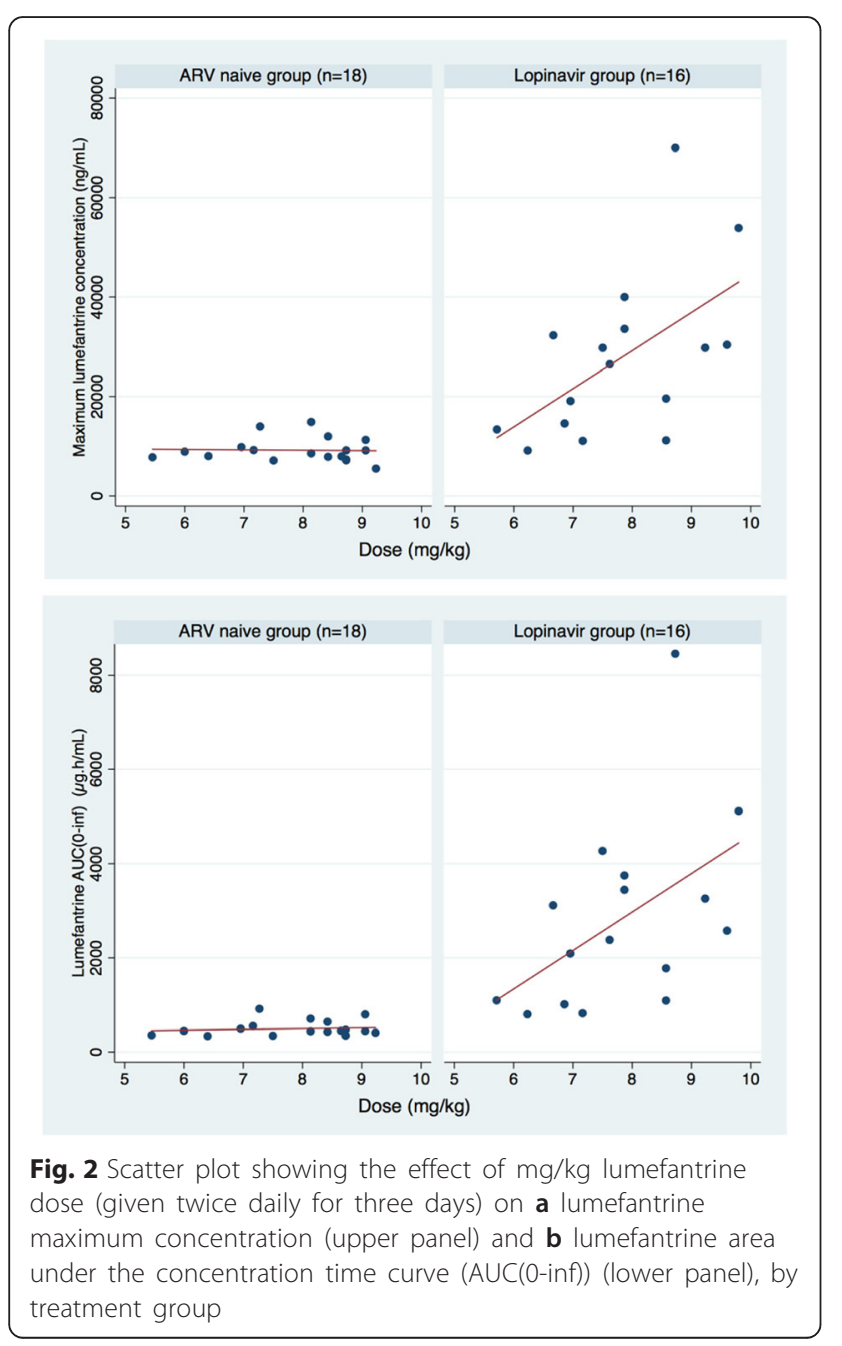

elimination half-life (Table 3, Figs. 3 and 4). These findings were confirmed in the mixed-effect model, which also showed that the dihydroartemisinin AUC increased significantly between the first and last dose in the ARV-naïve group (GMR 1.30 [95 \% CI 1.03-1.64], $p=0.03$ ) but not in the lopinavir group $(p=0.195)$ (Table 4$)$.

\section{Electrocardiograph safety}

The QTcF intervals were similar between treatment groups at the predicted time to maximum concentration ( Tmax, $68 \mathrm{~h}$ after the first dose, which was very close to the observed Cmax of $67 \mathrm{~h}$ in the lopinavir group) and within groups between screening and $\sim$ Tmax. There were no QTcF intervals $>450 \mathrm{msec}$, even at $\sim$ Tmax. The median (range) QTcF intervals were 406 (370-443) ms at $\sim$ Tmax in the lopinavir group, compared with 409 (366-436) $\mathrm{ms}$ in the ARV-naïve group $(p=0.72)$ at $68 \mathrm{~h}$, and $408(370-438) \mathrm{ms}$ in the lopinavir group at screening $(p=0.89)$. PR intervals were not prolonged in any participant, but did increase slightly in both groups between screening and $\sim \operatorname{Tmax}$ (mean from 161 to $168 \mathrm{~ms}$ in ARV-naïve group $(p=0.027)$ and from 152 to $166 \mathrm{~ms}$ in the lopinavir group $(p=0.012)$. However, PR intervals were similar between treatment groups at screening $(p=0.41)$ and $\sim \operatorname{Tmax}(p=0.62)$. Lumefantrine concentrations at $\sim$ Tmax were not associated with QTcF $(p=0.54)$ or PR intervals $(p=0.12)$.

\section{Adverse events}

There were 173 adverse events recorded overall, with no serious or severe adverse events. All patients except one in the ARV-naive group experienced at least one adverse event. Within 21 days of starting the full (6-dose) artemether-lumefantrine treatment there were 42 adverse events occurring in the lopinavir group $(n=16)$ and 35 adverse events in the ARV-naïve group $(n=18)$ 

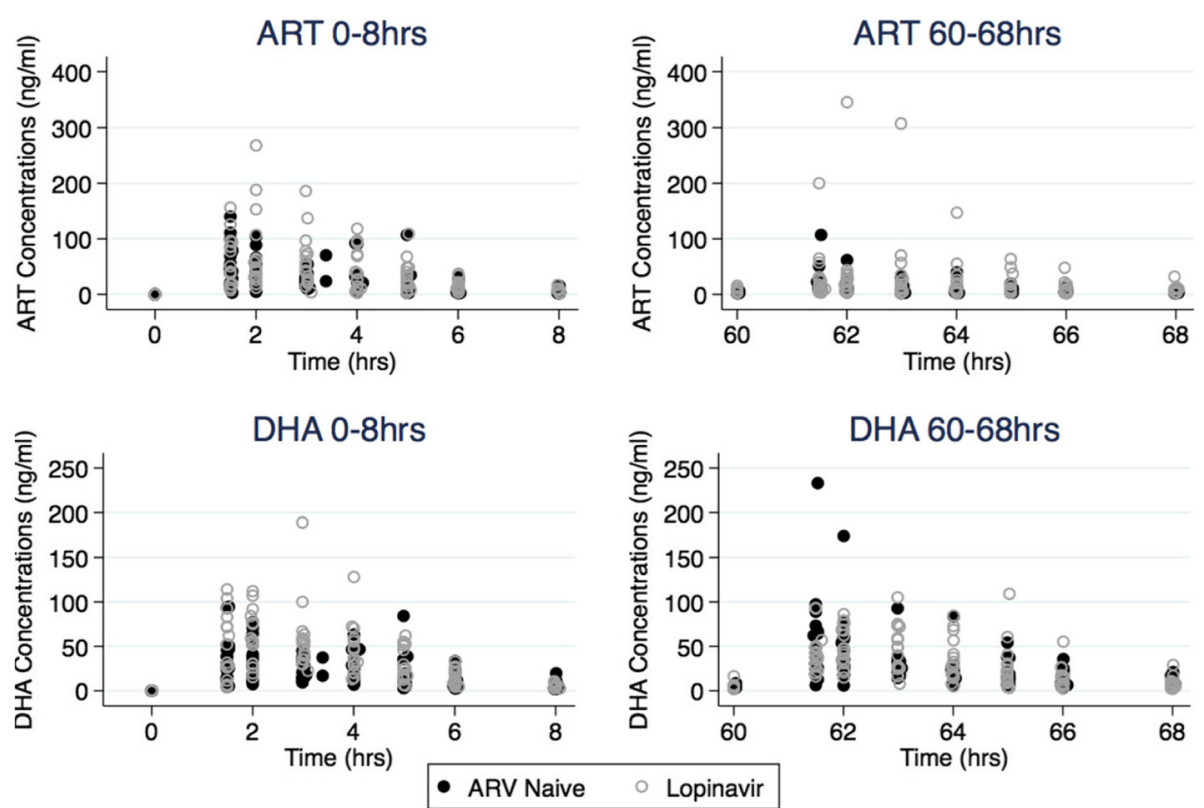

Fig. 3 Scatter plot of Plasma artemether (ART) and dihydroartemisinin (DHA) concentrations over time, by study group and treatment period (after dose 1 (0-8 hours), and after dose 6 (60-68 hours))

[Table 5]. Of these adverse events, 27 were considered possibly related to study drug, 15 and 12 in the ARVnaïve and lopinavir groups, respectively $(p=0.21)$. Most events were classified as mild, and all four of the moderate adverse events were not considered related to artemether-lumefantrine. The most common adverse events considered possibly related to artemetherlumefantrine were: headache $(n=7$, with 4 in ARV-naïve group and 3 in lopinavir group), nausea ( $n=5$, with 3 in ARV-naïve group and 2 in lopinavir group), diarrhoea ( $n=3$, with 1 in ARV-naïve group and 2 in lopinavir group), and flatulence ( $n=3$, all in ARV-naïve group).
Among those in the lopinavir group given a single dose of artemether-lumefantrine during Phase $1 \quad(n=18)$, there were 37 treatment emergent adverse events, seven of which were considered possibly related to artemetherlumefantrine (Additional file 1$)$, with a trend $(p=0.095)$ towards fewer adverse reactions than in those given the full 6-dose artemether-lumefantrine treatment, regardless of the antiretroviral regimen used. A further $11 \mathrm{AEs}$ were reported before the artemether-lumefantrine dose was administered, and another 27 after 21 days of follow up (mostly between the single and multiple artemetherlumefantrine dose phases in the lopinavir group). Another

Table 3 Artemether and dihydroartemisinin pharmacokinetic parameters in HIV-1 infected patients who are antiretroviral (ARV)-naïve or on lopinavir-based antiretroviral therapy (ART), after artemether-lumefantrine dose 1 (0-8 hours) and dose 6 (60-68 hours)

\begin{tabular}{|c|c|c|c|c|c|c|}
\hline \multicolumn{4}{|l|}{$0-8$ hours } & \multicolumn{3}{|l|}{$60-68$ hours } \\
\hline & ARV-Naïve group & Lopinavir group & $p$ value & ARV-Naïve group & Lopinavir group & $p$ value \\
\hline \multicolumn{7}{|l|}{ Artemether } \\
\hline$C_{\max }(n g / m L)$ & $59.7(37.8-88.9)$ & $85.8(39.7-145)$ & 0.16 & $11.9(8.2-17.5)$ & $16.5(7.2-50.5)$ & 0.35 \\
\hline $\mathrm{T}_{\max }(\mathrm{h})$ & $1.5(1.5-2.0)$ & $1.8(1.5-3.0)$ & 0.97 & $61.5(61.5-62.0)$ & $61.5(61.5-62.0)$ & 0.84 \\
\hline $\mathrm{AUC}_{(0-\mathrm{inf})}(\mathrm{ng} \cdot \mathrm{h} / \mathrm{mL})$ & $151.0(110.7-220.6)$ & $220.0(113.9-431.2)$ & 0.17 & $71.1(45.5-114.2)$ & $93.8(37.5-219.1)$ & 0.37 \\
\hline $\mathrm{t}_{1 / 2}(\mathrm{hr})$ & $1.5(1.1-1.7)$ & $1.4(0.9-1.6)$ & 0.39 & $2.9(1.8-5.4)$ & $3.0(1.9-3.4)$ & 0.68 \\
\hline \multicolumn{7}{|l|}{ Dihydroartemisinin } \\
\hline$C_{\max }(n g / m L)$ & $42.2(31.8-63.1)$ & $77.5(59.4-102)$ & 0.004 & $40.0(31.2-66.7)$ & $65.8(38.2-92.7)$ & 0.21 \\
\hline $\mathrm{T}_{\max }(\mathrm{h})$ & $2.0(1.5-4.0)$ & $2.0(1.5-3.0)$ & 0.41 & $61.5(61.5-62.0)$ & $61.6(61.5-62.5)$ & 0.71 \\
\hline$A \cup C_{(0-\mathrm{inf})}(\mathrm{ng} \cdot \mathrm{h} / \mathrm{mL})$ & $123.8(101.3-235.6)$ & $283.6(178.1-340.7)$ & 0.001 & $165.7(143.7-246.5)$ & $243.5(145.1-305.0)$ & 0.27 \\
\hline$T_{1 / 2}(h)$ & $1.6(1.3-2.1)$ & $1.4(1.1-1.6)$ & 0.07 & $2.0(1.8-2.7)$ & $1.8(1.5-2.0)$ & 0.02 \\
\hline
\end{tabular}

Values are reported as median (interquartile range [IQR]). Cmax, maximal concentration; Tmax, time at the maximal concentration; AUC (0-inf), area under the plasma concentration-time curve, from $0 \mathrm{~h}$ to infinity; $t 1 / 2$, elimination half-life. Statistical significance ( $\mathrm{p}$ values) calculated using the Kruskal-Wallis test 


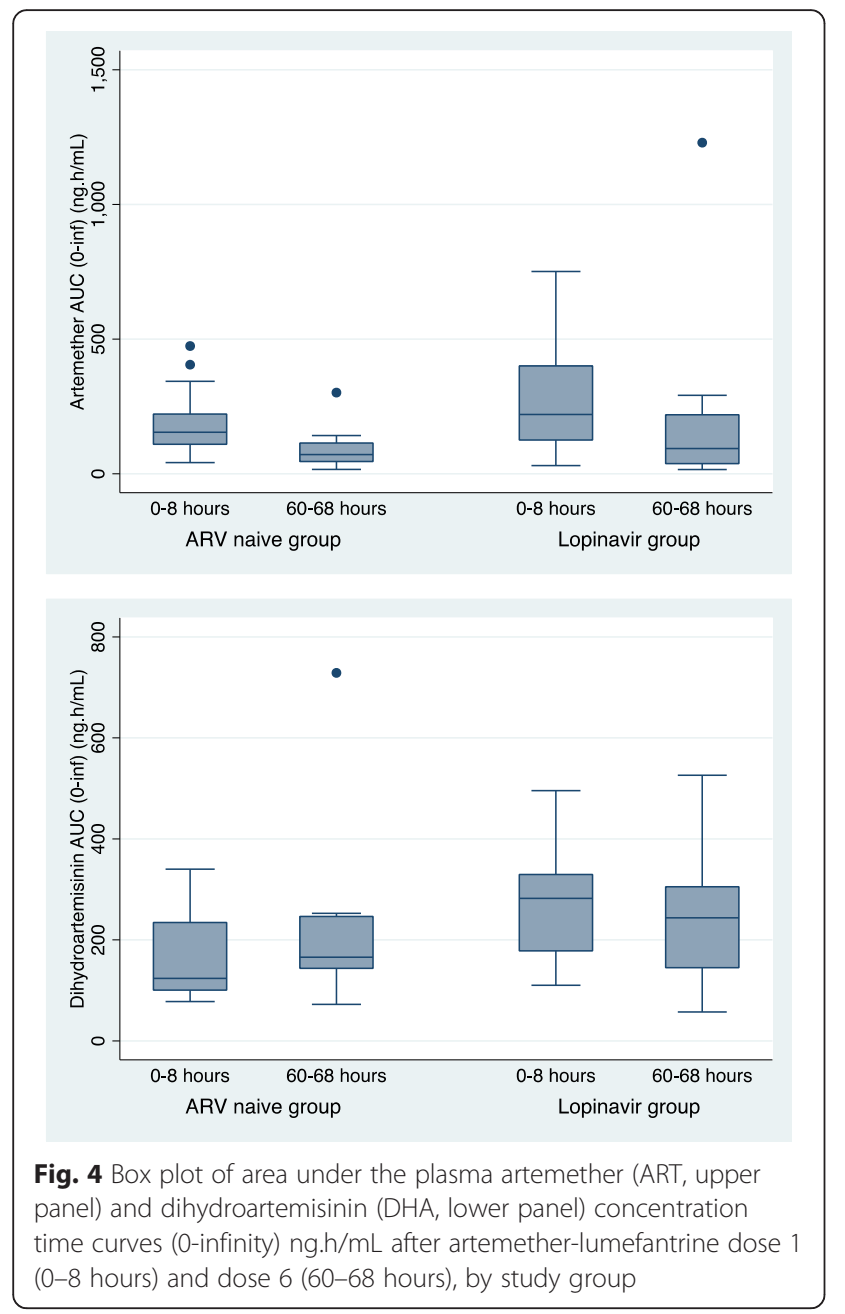

21 adverse events were detected in a methods sub-study with more intensive enquiry about adverse events using checklists, in depth interviews and focus group discussions only conducted in some participants in the lopinavir group [22].

\section{Discussion}

We investigated the safety and pharmacokinetics of artemether-lumefantrine after the recommended six-dose regimen in HIV-infected adult patients, comparing results in ARV-naïve patients with those on lopinavirbased antiretroviral therapy. We found that those on antiretroviral therapy had a 10-fold increase in their day-7 lumefantrine concentrations, with an almost five-fold increase in their lumefantrine $\mathrm{AUC}_{(0-\text { inf })}$ and almost three-fold increase in the maximum lumefantrine concentration. Despite this substantially elevated exposure, detailed assessments for clinical, haematological, biochemical or electrocardiographic adverse events raised no safety concerns associated with concomitant artemether-lumefantrine and lopinavir-based ART administration. There were no serious adverse events, and most adverse events were mild in intensity. Within 21 days of starting artemether-lumefantrine there were similar numbers of treatment emergent adverse events (42 vs. 35$)$ and adverse reactions (12 vs. 15$)$ in the lopinavir and ARV-naïve groups, respectively.

Lumefantrine is chemically similar to halofantrine, which is known to cause significant QT prolongation and cardiac arrhythmias even at standard doses. This structural similarity initially raised the concern of potential cardiac toxicity, but this was not confirmed in a prospective study [23]. The electrocardiographic assessments in the lopinavir group, including those at the time of predicted maximal lumefantrine concentration, did not show prolonged PR or QTcF intervals, and were not significantly different from the intervals found in the ARV-naïve group. This is consistent with findings by Byakika-Kibwika et al. who assessed cardiac conduction safety in HIV-positive adults, and found that QTc intervals remained well within the normal limits over the $72 \mathrm{~h}$ after a single artemether-lumefantrine dose, although the mean QTc interval after AL administration was longer in the lopinavir arm compared to the ARVnaïve arm [24].

Although artemether pharmacokinetic parameters were not significantly different between treatment groups in either period, we found a significant doseoccasion effect with five-fold decreases in artemether maximal concentrations, and more than two-fold decreases in AUC between the first and last dose. This is expected given the auto-induction previously described with the artemisinins $[16,25-28]$. The dihydroartemisinin

Table 4 Mixed-effects model of the effects of dose occasion, treatment group and covariates on dihydroartemisinin exposure in HIV-1 infected patients

\begin{tabular}{lll}
\hline Dose occasion effect (last/first dose occasion) & AUC GMR (95 \% Cl) & $C_{\max }$ GMR (95 \% Cl) \\
- Antiretroviral naïve group & $1.30(1.03-1.64)$ & $1.12(0.84-1.49)$ \\
- Lopinavir group & $0.84(0.65-1.09)$ & $0.77(0.56-1.05)$ \\
Treatment group effect (lopinavir group/naïve group) & $1.73(1.26-2.37)$ & $1.73(1.24-2.43)$ \\
-0 to $8 \mathrm{~h}$ & $1.12(0.56-1.55)$ & $1.19(0.58-2.57)$ \\
\hline
\end{tabular}


Table 5 Treatment Emergent Adverse Events by treament group, causality and intensity

\begin{tabular}{|c|c|c|c|c|c|c|c|c|}
\hline & \multicolumn{4}{|c|}{ ARV naïve group ( $n=18)$} & \multicolumn{4}{|c|}{ Lopinavir group ( $n=16)$} \\
\hline & \multicolumn{2}{|c|}{ AL suspected } & \multicolumn{2}{|c|}{ AL not suspected } & \multicolumn{2}{|c|}{ AL suspected } & \multicolumn{2}{|c|}{ AL not suspected } \\
\hline & Mild & Moderate & Mild & Moderate & Mild & Moderate & Mild & Moderate \\
\hline \multicolumn{9}{|l|}{ Gastrointestinal disorders } \\
\hline Abdominal pain & & & 1 & & & & & \\
\hline Constipation & & & & & & & 1 & \\
\hline Decreased appetite & 1 & & 1 & & & & & \\
\hline Diarrhoea & 1 & & 1 & & 2 & & 1 & \\
\hline Dyspepsia & 2 & & & & & & & \\
\hline Epigastric discomfort & & & 1 & & 1 & & & \\
\hline Flatulence & 3 & & & & & & & \\
\hline Gastrooesophageal reflux disease & & & & & & & 1 & \\
\hline Nausea & 3 & & & & 2 & & & \\
\hline Vomiting & & & & & 2 & & & \\
\hline \multicolumn{9}{|l|}{ Infections and infestations } \\
\hline Gingivitis & & & & & & & 1 & \\
\hline Influenza-like illness & & & 3 & & & & 2 & \\
\hline \multicolumn{9}{|l|}{ Nervous system disorders } \\
\hline Dizziness & 1 & & & & 1 & & & \\
\hline Headache & 4 & & 3 & 1 & 3 & & 2 & \\
\hline Presyncope & & & & & & & & 1 \\
\hline \multicolumn{9}{|c|}{ Respiratory, thoracic and mediastinal disorders } \\
\hline Nasal congestion & & & & & & & 1 & \\
\hline Rhinorrhoea & & & & & & & 1 & \\
\hline \multicolumn{9}{|l|}{ Skin and subcutaneous tissue disorders } \\
\hline Abscess & & & & & & & 1 & \\
\hline Body tinea & & & 1 & & & & & \\
\hline Rash & & & 1 & & & & 1 & \\
\hline Seborrhoeic dermatitis & & & & & & & 1 & \\
\hline \multicolumn{9}{|c|}{ General disorders and administration site conditions } \\
\hline Fatigue & & & & & 1 & & 1 & \\
\hline Injection site reaction & & & 2 & & & & 3 & \\
\hline \multicolumn{9}{|c|}{ Musculoskeletal and connective tissue disorders } \\
\hline Back / neck pain & & & & & & & 3 & \\
\hline Muscle twitching & & & 2 & & & & & \\
\hline \multicolumn{9}{|l|}{ Renal and urinary disorders } \\
\hline Frequency of micturition & & & & & & & 1 & \\
\hline Urinary tract infection & & & 1 & & & & 1 & \\
\hline \multicolumn{9}{|c|}{ Reproductive system and breast disorders } \\
\hline Vaginal discharge & & & 1 & & & & & \\
\hline \multicolumn{9}{|l|}{ Immune system disorders } \\
\hline Seasonal allergy & & & & & & & 1 & \\
\hline \multicolumn{9}{|l|}{ Vascular disorders } \\
\hline Epistaxis & & & & & & & 1 & \\
\hline Hypertension & & & 1 & & & & 1 & \\
\hline
\end{tabular}


Table 5 Treatment Emergent Adverse Events by treament group, causality and intensity (Continued)

\begin{tabular}{l}
\hline Eye disorders \\
Uveitis \\
Psychiatric disorders \\
Alcoholic hangover \\
Self-induced vomiting \\
Metabolism and nutrition disorders \\
Hypercholesterolaemia \\
Oedema peripheral \\
Injury, poisoning and procedural complications \\
Skin wounds
\end{tabular}

maximal concentration and exposure were almost double in the lopinavir group compared to the ARV-naïve group at 0 to $8 \mathrm{~h}$, although these were similar between treatment groups after the last dose. Artemether and dihydroartemisinin exposure in our ARV-naïve group were similar to the results from previously published healthy volunteer studies, suggesting that there is not a marked HIV disease effect $[19,29]$. Unlike the findings in a single artemetherlumefantrine dose study in HIV-infected Ugandan adults [30], our dihydroartemisinin concentrations were higher in the lopinavir group at 0-8 hours, which was also reported by German et al. in healthy volunteers [29]. If confirmed, this increased artemisinin exposure with lopinavir/ritonavir may result in some benefit, particularly in the light of confirmed artemisinin resistance having spread from western Cambodia across mainland South East Asia, from southern Vietnam to western Myanmar [31-33].

Two previous studies in American and Ugandan adults have examined the interaction of lopinavir-based antiretroviral therapy and artemether-lumefantrine, using different methods to our study [25, 29]. Due to concerns about substantially elevated lumefantrine concentrations we included a single-dose phase, which then determined the dosing regimen used for our second six-dose phase. Our single-dose phase found a doubling of the lumefantrine exposure, which was similar to the result found in a single-dose study of Ugandan malaria-negative, HIVinfected patients on lopinavir-based antiretroviral therapy, and in a healthy volunteer, six-dose study conducted in the United States (Table 6). In the six-dose phase of our trial, the lumefantrine exposures in our ARV-naïve group were similar to those in American healthy volunteers (445 vs. 456 ug.h/mL respectively). However, our patients on lopinavir-based antiretroviral therapy had 1.6-fold higher maximal concentrations and 2.3-fold higher exposures than the healthy volunteers on lopinavir in the American study. Hoglund et al. (2015) who reanalysed the pharmacokinetic data from the singledose study in Ugandan HIV-infected, malaria-negative adults [30] using non-linear mixed-effect modelling, also found that lopinavir-ritonavir increased lumefantrine exposure by $439 \%$, largely explained by its clearance being decreased by $62 \%$.

To minimise any safety risks, and to obtain an estimate of the effect size of the pharmacokinetic drugdrug interaction without confounding by any malaria disease effect, neither our study nor both studies in adults cited above included malaria patients [25, 29]. Thus, we could not determine whether increased lumefantrine exposure improved antimalarial therapeutic efficacy. Previous studies in malaria patients have shown that the day-7 lumefantrine concentration is the most important single concentration measure in terms of its correlation with the area under the concentration time curve and its association with treatment response $[4,11,34]$. In a large pooled analysis in 2528 patients, treatment failure was associated with low day-7 lumefantrine concentrations; the risk of recrudescence decreased by $36 \%$ (HR $0.64(0.55$ to 0.74$)<0.001)$ and the risk of reinfection decreased by $21 \%$ (HR 0.79 ( 0.72 to $0.87)<0.001$ ) with a doubling of lumefantrine concentrations [11]. Thus the marked increases in lumefantrine concentration observed in our lopinavir group would be expected to reduce their risk of treatment failure. This has been evaluated in malaria and HIV co-infected children, but not yet in co-infected adults [35, 36]. In co-infected Ugandan children under six-years of age (median age 2.9 years), recurrent malaria and malaria incidence were lower following artemether-lumefantrine treatment in those on lopinavir-based ART than in those on nonnucleoside reverse transcriptase inhibitor (NNRTI)based ART. The Ugandan paediatric lopinavir group had elevated day-7 lumefantrine concentrations of $388 \mathrm{ng} / \mathrm{mL}$ and day-7 concentrations above $300 \mathrm{ng} / \mathrm{mL}$ 
Table 6 Drug interaction studies between artemether-lumefantrine and lopinavir/ritonavir, showing median lumefantrine, artemether and dihydroartemisinin pharmacokinetic parameters with and without lopinavir/ritonavir

\begin{tabular}{|c|c|c|c|c|c|c|c|c|c|c|c|c|}
\hline \multirow{2}{*}{$\begin{array}{l}\text { Study } \\
\text { (Reference) }\end{array}$} & \multirow{2}{*}{ Population } & \multirow[t]{2}{*}{ Comparator groups } & \multirow{2}{*}{$\begin{array}{l}\text { Artemether- } \\
\text { lumefantrine } \\
\text { dose }\end{array}$} & \multirow{2}{*}{$\begin{array}{l}\text { Concomitant } \\
\text { fat intake }\end{array}$} & \multirow{2}{*}{$\begin{array}{l}\text { Samples per } \\
\text { participant } \\
\text { (matrix) }\end{array}$} & \multicolumn{3}{|l|}{ Lumefantrine } & \multicolumn{2}{|l|}{ Artemether } & \multicolumn{2}{|l|}{ Dihydro-artemisinin } \\
\hline & & & & & & $\mathrm{AUC}$ & Cmax & Day 7 & $\mathrm{AUC}$ & Cmax & AUC & Cmax \\
\hline \multirow[t]{2}{*}{ Kredo } & \multirow[t]{2}{*}{$\begin{array}{l}\text { HIV infected, } \\
\text { malaria negative } \\
\text { adults }\end{array}$} & \multirow[t]{2}{*}{$\begin{array}{l}\text { Parallel: Lopinavir } \\
\text { based ART vs. } \\
\text { ARV-naïve }\end{array}$} & $\begin{array}{l}\text { Single dose } \\
\text { (Phase 1) }\end{array}$ & Yes & 22 (Plasma) & $\begin{array}{l}\text { Single dose: } \\
\text { AUC }_{(0 \text {-inf) }} 1852 \\
\text { vs. } 1133 \mu \mathrm{g} . \mathrm{h} / \mathrm{mL}\end{array}$ & $\begin{array}{l}5.26 \mathrm{vs} . \\
2.50 \mu \mathrm{g} / \mathrm{mL}\end{array}$ & NA & NA & NA & NA & NA \\
\hline & & & $\begin{array}{l}\text { Standard 6-dose } \\
\text { regimen (Phase 2) }\end{array}$ & Yes & 33 (Plasma) & $\begin{array}{l}\mathrm{AUC}_{(0-\mathrm{inf})} 2477 \\
\text { vs. } 445 \mu \mathrm{g} \cdot \mathrm{h} / \mathrm{mL}\end{array}$ & $\begin{array}{l}28.15 \mathrm{vs} \\
8.76 \mu \mathrm{g} / \mathrm{mL}\end{array}$ & $\begin{array}{l}3170 \mathrm{vs} . \\
336 \mathrm{ng} / \mathrm{mL}\end{array}$ & $\begin{array}{l}\mathrm{AUC}_{(0-8 \mathrm{~g})} 220 \\
\text { vs. } 151 \mathrm{ng} \cdot \mathrm{h} / \mathrm{mL}\end{array}$ & $\begin{array}{l}85.8 \mathrm{vs.} \\
59.7 \mathrm{ng} / \mathrm{mL}\end{array}$ & $\begin{array}{l}\mathrm{AUC}_{(0-8 \mathrm{~h})} 283.6 \\
\text { vs. } 123.8 \mathrm{ng} . \mathrm{h} / \mathrm{mL}\end{array}$ & $\begin{array}{l}77.5 \mathrm{vs.} \\
42.2 \mathrm{ng} / \mathrm{mL}\end{array}$ \\
\hline $\begin{array}{l}\text { Byakika- } \\
\text { Kibwika }^{25}\end{array}$ & $\begin{array}{l}\text { HIV infected, } \\
\text { malaria negative } \\
\text { adults }\end{array}$ & $\begin{array}{l}\text { Parallel: Lopinavir } \\
\text { based ART vs. } \\
\text { ARV- naïve }\end{array}$ & Single dose & Yes & 9 (Plasma) & $\begin{array}{l}\text { AUC }(0-\text {-inf } 267 \\
\text { vs. } 47 \mu \mathrm{g} . \mathrm{h} / \mathrm{mL}\end{array}$ & $\begin{array}{l}7.10 \mathrm{vs} . \\
2.53 \mu \mathrm{g} / \mathrm{mL}\end{array}$ & NA & $\begin{array}{l}\text { AUC }_{(0-i n f)} 162 \\
\text { vs } 271 \mathrm{ng} . \mathrm{h} / \mathrm{mL}\end{array}$ & $\begin{array}{l}56 \mathrm{vs} \\
112 \mathrm{ng} / \mathrm{mL}\end{array}$ & $\begin{array}{l}\text { AUC }_{(0-\text { inf })} 180 \\
\text { vs } 217 \mathrm{ng} \cdot \mathrm{h} / \mathrm{mL}\end{array}$ & $\begin{array}{l}73 \mathrm{vs} \\
66 \mathrm{ng} / \mathrm{mL}\end{array}$ \\
\hline German $^{29}$ & $\begin{array}{l}\text { Healthy adult } \\
\text { volunteers }\end{array}$ & $\begin{array}{l}\text { Cross-over: Artemether } \\
\text { lumefantrine given } \\
\text { before and after } 26 \\
\text { days Lopinavir-based } \\
\text { ART }\end{array}$ & $\begin{array}{l}\text { Standard } 6 \text { dose } \\
\text { regimen }\end{array}$ & Yes & 10 (Plasma) & $\begin{array}{l}\mathrm{AUC}_{(0-264)} 1073 \\
\text { vs } 456\end{array}$ & $\begin{array}{l}17.4 \mathrm{vs} \\
12.5 \mu \mathrm{g} / \mathrm{mL}\end{array}$ & NA & $\begin{array}{l}A \cup C_{(0-i n f)} 40.5 \\
\text { vs. } 62.0 \mathrm{ng} \cdot \mathrm{h} / \mathrm{mL}\end{array}$ & $\begin{array}{l}11.2 \mathrm{vs} \\
14.3 \mathrm{ng} / \mathrm{mL}\end{array}$ & $\begin{array}{l}\text { AUC }_{(0-\text { inf) }} 109 \\
\text { vs } 198 \text { ng.h/mL }\end{array}$ & $\begin{array}{l}37.3 \mathrm{vs} \\
58.8 \mathrm{ng} / \mathrm{mL}\end{array}$ \\
\hline \multirow[t]{3}{*}{ Achan $^{35}$} & \multirow{3}{*}{$\begin{array}{l}\text { HIV-infected } \\
\text { children with } \\
\text { malaria on ART }\end{array}$} & \multirow{3}{*}{$\begin{array}{l}\text { Parallel: Lopinavir- } \\
\text { based ART vs. NRTI- } \\
\text { based antiretrovirals }\end{array}$} & \multirow[t]{3}{*}{$\begin{array}{l}\text { Standard } 6 \text { dose } \\
\text { regimen }\end{array}$} & \multirow[t]{3}{*}{$\begin{array}{l}\text { Not } \\
\text { reported }\end{array}$} & \multirow[t]{3}{*}{$\begin{array}{l}1 \\
\text { (Capillary blood) }\end{array}$} & \multirow[t]{3}{*}{ NA } & \multirow[t]{3}{*}{ NA } & $\begin{array}{l}\text { Lopinavir: } \\
926 \text { ng/mL }\end{array}$ & \multirow[t]{3}{*}{ NA } & \multirow[t]{3}{*}{ NA } & \multirow[t]{3}{*}{ NA } & \multirow[t]{3}{*}{ NA } \\
\hline & & & & & & & & $\begin{array}{l}\text { Nevirapine: } \\
388 \text { ng/mL }\end{array}$ & & & & \\
\hline & & & & & & & & $\begin{array}{l}\text { Efavirenz: } \\
97 \mathrm{ng} / \mathrm{mL}\end{array}$ & & & & \\
\hline
\end{tabular}


were associated with a significantly decreased risk of malaria recurrence within 63 days.

Previous studies show that the absorption of lumefantrine was close to saturated at the currently recommended dose [37], which is a major obstacle for optimising dosage recommendations for patient sub-groups who do not achieve target concentrations with the currently recommended dosage regimens [12]. However, we showed that an increase in lumefantrine $\mathrm{mg} / \mathrm{kg}$ dose was associated with a significant increase in the lumefantrine Cmax and $\mathrm{AUC}_{(0 \text {-inf })}$, in the lopinavir group but not the ARV-naïve group. Lumefantrine is $\mathrm{N}$-debutylated by CYP3A4, but desbutyl-lumefantrine represents approximately $0.3-1 \%$ of the parent exposure [12] suggesting inhibition of intestinal CYP3A4 and possibly other transporters as a mechanism. These findings may contribute towards a better understanding of the mechanism underlying the nonlinear relationship between lumefantrine dose and bioavailability, and of interventions that could be studied in key target populations in whom lumefantrine is currently sub-optimally dosed.

\section{Conclusions}

Despite markedly higher lumefantrine exposure, intensive monitoring in our relatively small study raised no safety concerns associated with using the current recommended six-dose regimen of artemether-lumefantrine in HIV-infected adult patients receiving lopinavir-based antiretroviral therapy. Elevated lumefantrine concentrations have been shown to reduce the risk of treatment failure as reported previously in malaria patients of all ages $[4,11]$, and in malaria and HIV co-infected children $[35,36]$. Further evidence in adults co-infected with malaria and HIV is required to substantiate these results. Our ARV-naïve patients confirmed previous studies' findings that lumefantrine absorption is close to saturation with currently recommended doses, but this doselimited absorption was overcome in those on lopinavirbased ART.

\section{Additional file}

Additional file 1: Treatment Emergent Adverse Events within 21 days after a single artemether-lumefantrine dose in 18 HIV-infected adults on lopinavir-based antiretrovirals, by causality and intensity. (DOCX $13 \mathrm{~kb})$

\section{Competing interests}

The authors declare that they have no potential conflicts of interest that may have unduly influenced the results of this study.

\section{Authors' contributions}

$\mathrm{KB}$ conceived and designed the study with input from GM, KC and JS; TK and KB drafted the manuscript; KM conducted statistical analyses and reviewed manuscript for accuracy; LW led data management; LW and PS are laboratory partner's responsible for sample analysis. KB, JS, TK, KC,
GM, LW, PS contributed to conduct of study and all authors approved the final manuscript.

\section{Acknowledgments}

This investigator-initiated study was funded by the Haughton Institute, which is funded through the Global Health Research Board from the Irish Department of Foreign Affairs and by the ACT Consortium, which is funded through a grant from the Bill and Melinda Gates Foundation to the London School of Hygiene and Tropical Medicine.

We gratefully acknowledge the contributions of the study patients for their time and patience; the SEACAT evaluation team, in particular, Rae Thomas, Liz Allen and Ludwig Heiberg; and, the invaluable guidance provided by the Data Safety Monitoring Board comprising Anton Pozniak (chair), Marta Boffito (both Chelsea and Westminster Hospital, London, UK), Piero Olliaro (WHO TDR, Geneva, Switzerland), Bill Burman (University of Colorado, Denver, USA) and Bonnie Cundill (London School of Hygiene and Tropical Medicine, London, UK).

\section{Data access statement}

Complete anonymised individual patient data has been contributed to the WorldWide Antimalarial Resistance Network (WWARN) data repository. Third party access to this data can be requested by submitting an analysis proposal to WWARN (info@wwarn.org).

\section{Author details}

${ }^{1}$ Division of Clinical Pharmacology, Department of Medicine, University of Cape Town, Cape Town, South Africa. ${ }^{2}$ Cochrane South Africa, South African Medical Research Council, Cape Town, South Africa. ${ }^{3}$ Department of Statistical Sciences, University of Cape Town, Cape Town, South Africa.

${ }^{4}$ WorlldWide Antimalarial Resistance Network (WWARN), Oxford, UK.

Received: 31 August 2015 Accepted: 12 January 2016

Published online: 27 January 2016

\section{References}

1. World Health Organization. World Malaria Report 2014. Switzerland: World Health Organization. [Available from: http://www.who.int/malaria/ publications/world_malaria_report_2014/wmr-2014-no-profiles.pdf].

2. White NJ, Stepniewska K, Barnes K, Price RN, Simpson J. Simplified antimalarial therapeutic monitoring: using the day-7 drug level? Trends Parasitol. 2008;24(4):159-63.

3. Barnes Kl, Lindegardh N, Ogundahunsi O, Olliaro P, Plowe CV, Randrianarivelojosia $\mathrm{M}$, et al. World Antimalarial Resistance Network (WARN) IV: clinical pharmacology. Malar J. 2007;6:122.

4. White NJ, van Vugt M, Ezzet F. Clinical pharmacokinetics and pharmacodynamics and pharmacodynamics of artemether-lumefantrine. Clin Pharmacokinet. 1999:37(2):105-25.

5. Ezzet F, van Vugt M, Nosten F, Looareesuwan S, White NJ. Pharmacokinetics and pharmacodynamics of lumefantrine (benflumetol) in acute falciparum malaria. Antimicrob Agents Chemother. 2000;44(3):697-704.

6. McGready R, Tan SO, Ashley EA, Pimanpanarak M, Viladpai-Nguen J, Phaiphun $L$, et al. A randomised controlled trial of artemether-lumefantrine versus artesunate for uncomplicated plasmodium falciparum treatment in pregnancy. PLoS Med. 2008;5(12):e253.

7. Checchi F, Piola P, Fogg C, Bajunirwe F, Biraro S, Grandesso F, et al. Supervised versus unsupervised antimalarial treatment with six-dose artemether-lumefantrine: pharmacokinetic and dosage-related findings from a clinical trial in Uganda. Malar J. 2006:5:59.

8. Rahman MM, Dondorp AM, Day NP, Lindegardh N, Imwong M, Faiz MA, et al. Adherence and efficacy of supervised versus non-supervised treatment with artemether/lumefantrine for the treatment of uncomplicated Plasmodium falciparum malaria in Bangladesh: a randomised controlled trial. Trans R Soc Trop Med Hyg. 2008;102(9):861-7.

9. Price RN, Uhlemann AC, van Vugt M, Brockman A, Hutagalung R, Nair S, et al. Molecular and pharmacological determinants of the therapeutic response to artemether-lumefantrine in multidrug-resistant Plasmodium falciparum malaria. Clin Infect Dis. 2006;42(11):1570-7.

10. Denis MB, Tsuyuoka R, Lim P, Lindegardh N, Yi P, Top SN, et al. Efficacy of artemether-lumefantrine for the treatment of uncomplicated falciparum malaria in northwest Cambodia. Trop Med Int Health. 2006;11(12):1800-7. 
11. WorldWide Antimalarial Resistance Network (WWARN) Lumefantrine PKV PD Study Group. Artemether-lumefantrine treatment of uncomplicated Plasmodium falciparum malaria: a systematic review and meta-analysis of day 7 lumefantrine concentrations and therapeutic response using individual patient data. BMC Med. 2015;13(1):227.

12. World Health Organization. Guidelines for the treatment of malaria. Switzerland: World Health Organization. 2015. 3rd edition: [Available from: http://www.who.int/malaria/publications/atoz/9789241549127/en/]

13. Oldfield V, Plosker GL. Lopinavir/Ritonavir: a review of its use in the management of HIV infection. Drugs. 2006;66(9):1275-99.

14. Khoo S, Back D, Winstanley P. The potential for interactions between antimalarial and antiretroviral drugs. AIDS. 2005;19(10):995-1005.

15. Flateau C, Le Loup G, Pialoux G. Consequences of HIV infection on malaria and therapeutic implications: a systematic review. Lancet Infect Dis. 2011; 11(7):541-56.

16. Kredo T, Mauff K, Van der Walt JS, Wiesner L, Maartens G, Cohen K, et al. Interaction between artemether-lumefantrine and nevirapine-based antiretroviral therapy in HIV-1-infected patients. Antimicrob Agents Chemother. 2011:55(12):5616-23.

17. South African National Department of Health. National antiretroviral treatment guidelines. 1st ed. Pretoria Jacana: Department of Health SA; 2004.

18. Lefevre G, Carpenter P, Souppart C, Schmidli H, Martin JM, Lane A, et al. Interaction trial between artemether-lumefantrine (Riamet) and quinine in healthy subjects. J Clin Pharmacol. 2002;42(10):1147-58.

19. Lefevre G, Carpenter P, Souppart C, Schmidli H, McClean M, Stypinski D. Pharmacokinetics and electrocardiographic pharmacodynamics of artemether-lumefantrine (Riamet) with concomitant administration of ketoconazole in healthy subjects. Br J Clin Pharmacol. 2002;54(5):485-92.

20. Goldenberg I, Moss AJ, Zareba W. QT interval: how to measure it and what is "normal". J Cardiovasc Electrophysiol. 2006;17(3):333-6.

21. Allen EN, Chandler Cl, Mandimika N, Pace C, Mehta U, Barnes Kl. Evaluating harm associated with anti-malarial drugs: a survey of methods used by clinical researchers to elicit, assess and record participant-reported adverse events and related data. Malar J. 2013;12:325.

22. Allen EN, Mushi AK, Massawe IS, Vestergaard LS, Lemnge M, Staedke SG, et al. How experiences become data: the process of eliciting adverse event, medical history and concomitant medication reports in antimalarial and antiretroviral interaction trials. BMC Med Res Methodol. 2013;13(1):140.

23. van Vugt M, Ezzet F, Nosten F, Gathmann I, Wilairatana P, Looareesuwan S, et al. No evidence of cardiotoxicity during antimalarial treatment with artemether-lumefantrine. Am J Trop Med Hyg. 1999;61(6):964-7.

24. Byakika-Kibwika P, Lamorde M, Lwabi P, Nyakoojo WB, Okaba-Kayom V, Mayanja-Kizza H, et al. Cardiac conduction safety during Coadministration of Artemether-lumefantrine and Lopinavir/Ritonavir in HIV-infected Ugandan adults. Chemother Res Pract. 2011;2011:393976.

25. Byakika-Kibwika P, Lamorde M, Okaba-Kayom V, Mayanja-Kizza H, Katabira E, Hanpithakpong W, et al. Lopinavir/ritonavir significantly influences pharmacokinetic exposure of artemether/lumefantrine in HIV-infected Ugandan adults. J Antimicrob Chemother. 2012;67(5):1217-23.

26. van Agtmael MA, Cheng-Qi S, Qing JX, Mull R, van Boxtel CJ. Multiple dose pharmacokinetics of artemether in Chinese patients with uncomplicated falciparum malaria. Int J Antimicrob Agents. 1999;12(2):151-8.

27. van Agtmael MA, Gupta V, van der Graaf CAA, van Boxtel CJ. The effect of grapefruit juice on the time-dependent decline of artemether plasma levels in healthy subjects[ast]. Clin Pharmacol Ther. 1999;66(4):408-14.

28. Svensson US, Alin H, Karlsson MO, Bergqvist Y, Ashton M. Population pharmacokinetic and pharmacodynamic modelling of artemisinin and mefloquine enantiomers in patients with falciparum malaria. Eur J Clin Pharmacol. 2002;58(5):339-51.

29. German P, Parikh S, Lawrence J, Dorsey G, Rosenthal PJ, Havlir D, et al. Lopinavir/ritonavir affects pharmacokinetic exposure of artemether/ lumefantrine in HIV-uninfected healthy volunteers. J Acquir Immune Defic Syndr. 2009;51(4):424-9.

30. Hoglund RM, Byakika-Kibwika P, Lamorde M, Merry C, Ashton M, Hanpithakpong W, et al. Artemether-lumefantrine co-administration with antiretrovirals: population pharmacokinetics and dosing implications. $\mathrm{Br}$ J Clin Pharmacol. 2015;79(4):636-49.

31. Ashley EA, Dhorda M, Fairhurst RM, Amaratunga C, Lim P, Suon S, et al. Spread of artemisinin resistance in Plasmodium falciparum malaria. N Engl J Med. 2014;371(5):411-23.
32. World Health Organization. Emergency response to artemisinin resistance in the Greater Mekong subregion. Regional Framework for Action 2013-2015. 2013. Available from: http://www.who.int/malaria/publications/atoz/ 9789241505321/en/.

33. Tun KM, Imwong M, Lwin KM, Win AA, Hlaing TM, Hlaing T, et al. Spread of artemisinin-resistant Plasmodium falciparum in Myanmar: a cross-sectional survey of the K13 molecular marker. Lancet Infect Dis. 2015;15(4):415-21.

34. Mwesigwa J, Parikh S, McGee B, German P, Drysdale T, Kalyango JN et al. Pharmacokinetics of artemether-lumefantrine and artesunateamodiaquine in children in Kampala, Uganda. Antimicrob Agents Chemother. 2010;54(1):52-9.

35. Achan J, Kakuru A, Ikilezi G, Ruel T, Clark TD, Nsanzabana C, et al. Antiretroviral agents and prevention of malaria in HIV-infected Ugandan children. N Engl J Med. 2012;367(22):2110-8.

36. Kakuru A, Achan J, Muhindo MK, Ikilezi G, Arinaitwe E, Mwangwa F, et al. Artemisinin-based combination therapies are efficacious and safe for treatment of uncomplicated malaria in HIV-infected Ugandan children. Clin Infect Dis. 2014;59(3):446-53.

37. Ashley EA, Stepniewska K, Lindegardh N, McGready R, Annerberg A, Hutagalung $\mathrm{R}$, et al. Pharmacokinetic study of artemether-lumefantrine given once daily for the treatment of uncomplicated multidrug-resistant falciparum malaria. Trop Med Int Health. 2007;12(2):201-8.

\section{Submit your next manuscript to BioMed Central and we will help you at every step:}

- We accept pre-submission inquiries

- Our selector tool helps you to find the most relevant journal

- We provide round the clock customer support

- Convenient online submission

- Thorough peer review

- Inclusion in PubMed and all major indexing services

- Maximum visibility for your research

Submit your manuscript at www.biomedcentral.com/submit
Ciomed Central 